\title{
Rapid and Sensitive Identification of Epitope- Containing Peptides by Direct Matrix-Assisted Laser Desorption/Ionization Tandem Mass Spectrometry of Peptides Affinity-Bound to Antibody Beads
}

\author{
Christina S. Raska, Carol E. Parker, Susan W. Sunnarborg, \\ R. Marshall Pope, David C. Lee, Gary L. Glish, \\ and Christoph H. Borchers \\ University of North Carolina at Chapel Hill, Chapel Hill, North Carolina, USA
}

\begin{abstract}
A method has been developed for rapid and sensitive identification of epitope-containing peptides, based on direct MALDI-MS/MS analysis of epitope-containing peptides affinity bound to affinity beads. This technique provides sequence information of the epitope that allows unambiguous identification of the epitope either by database searching or de novo sequencing. With MALDI-MS, affinity beads with bound peptides can be placed directly on the MALDI target and analyzed. Coupling a MALDI source to an orthogonal injection quadrupole time-of-flight (QqTOF) mass spectrometer allows direct sequencing of the bound peptides. In contrast to ESI-MS/MS, elution of the affinity-bound peptides followed by additional concentration and purification steps is not required, thus reducing the potential for sample loss. Direct mass spectrometric sequencing of affinity-bound peptides eliminates the need for chemical or enzymatic sequencing. Other advantages of this direct MALDI-MS/MS analysis of epitope-containing peptides bound to the affinity beads include its sensitivity (femtomole levels) and speed. In addition, direct analysis of peptides on affinity beads does not adversely affect the high mass accuracy of a QqTOF, and database searching can be performed on the MS/MS spectra obtained. In proof-of-principle experiments, this method has been demonstrated on beads containing immobilized antibodies against phosphotyrosine, the c-myc epitope tag, as well as immobilized avidin. Furthermore, de novo sequencing of epitope-containing peptides is demonstrated. The first application of this method was with anti-FLAG-tag affinity beads, where direct MALDI MS/MS was used to determine an unexpected enzymatic cleavage site on a growth factor protein. (J Am Soc Mass Spectrom 2003, 14, 1076-1085) (c) 2003 American Society for Mass Spectrometry
\end{abstract}

$\mathrm{T}$ The determination of the amino acid sequence of epitope-containing peptides is of major interest in broad areas of biochemical and biomedical research. Information about the epitope and eventual cross-reactivity of the antibody is very important for quality assurance of antibodies, especially when these antibodies are to be used in diagnostic and protein chip technologies. In addition, knowledge about the epitopecontaining sequence also can provide crucial information for elucidating autoimmune diseases, as well as for

Published online August 4, 2003

Address reprint requests to Dr. C. Borchers, Department of Biochemistry and Biophysics, University of North Carolina, 402 Mary Ellen Jones Bldg. CB no. 7260, Chapel Hill, NC 27599-3290, USA. E-mail: christoph_borchers@ med.unc.edu

${ }^{*}$ Current address: Cirrus Pharmaceuticals, P.O. Box 14748, RTP, NC 27709, USA. proteomics and protein characterization studies. Some methods advocated for these studies utilize affinity chromatography techniques for isolating the epitopecontaining peptide(s). Identification of the affinity-isolated peptides can lead directly to identification of the protein and can also provide information on the location of modification sites, including phosphorylation sites.

For identification of epitope-containing peptides, mass spectrometry (MS) is advantageous because of its inherent speed, sensitivity, and its capability of sequencing peptides, compared to conventional methods $[1,2]$. Antibodies are immobilized on agarose beads, and are used for immunoprecipitation of epitope-containing peptides. Traditionally, solution-phase separations such as high performance liquid chromatography (HPLC) coupled to mass spectrometry or ionization 
techniques such as nano-electrospray mass spectrometry (nESI-MS) have been used to identify the epitopecontaining peptides. It is necessary, however, to elute the peptides from the beads prior to the analysis step, introducing the potential for sample loss. With matrixassisted laser desorption/ionization mass spectrometry (MALDI-MS) analysis, it is possible to perform MALDI-MS of affinity-bound peptides or proteins by spotting an aliquot of affinity beads directly onto the MALDI target, without prior elution.

It has been demonstrated for immobilized metal ion affinity chromatography (IMAC) [3], that the MALDI matrix solvent may actually strip the affinity-bound peptides or proteins off the beads during cocrystallization with the matrix. However, the term "direct" MALDI analysis is used for techniques where the affinity beads are placed directly on the target, to contrast this method with "indirect" methods involving separate and time-consuming purification/elution steps (with concomitant losses due to sample handling) prior to spotting the eluate on the target.

Until recently, only protein molecular weight information and peptide mass fingerprinting data could be obtained by direct MALDI-MS of affinity-bound analytes [4-6]. To obtain sequence information on a particular affinity-bound peptide, enzymatic sequencing experiments had to be performed while the peptides were still bound to the beads $[4,7,8]$. While this has proven to be a useful approach for epitope mapping and other applications $[6,8,9-11]$, the resulting data can be difficult to interpret if more than one peptide is isolated, or if several residues are cleaved simultaneously.

With the coupling of a MALDI source to quadrupole/time-of-flight instruments (QqTOF) [12, 13], sequencing can now be performed directly in the gas phase, via collision-induced dissociation (CID) with tandem mass spectrometry (MS/MS). The additional mass selection offers the advantages of directly obtaining sequence information from each peptide in a rapid manner, without interference from other bound peptides. Direct MALDI-MS/MS of phosphorylated peptides bound to IMAC beads on a QqTOF has previously been reported [14]. The work presented here extends the direct MALDI-MS/MS approach to sequence epitope-containing peptides bound to a variety of antibody beads. In contrast to earlier reports on MALDITOF instruments [4, 15], no loss of mass accuracy results from analyzing the affinity beads directly on the target. The data presented in this paper demonstrate that based on the high mass accuracy obtained with a QqTOF, database searching, and de novo sequencing for protein identification can be successfully performed on the MS/MS spectra obtained, even with only femtomoles of peptide present. Thus, the direct MALDIMS/MS approach for affinity-bound peptides is well suited to the rapid and sensitive sequencing of epitopecontaining peptides.

\section{Experimental}

\section{Materials}

The kinase domain of the insulin receptor peptide (KDIR) (Thr-Arg-Asp-Ile-Tyr-Glu-Thr-Asp-Tyr-Tyr-Arg-Lys), the phosphorylated kinase domain of the insulin receptor peptide no. 3 (pKDIR) (Thr-Arg-Asp-Ile-Tyr-Glu-ThrAsp-Tyr-pTyr-Arg-Lys), and the biotinylated kinase domain of the insulin receptor peptide (biotinKDIR) (BiotinThr-Arg-Asp-Ile-Tyr-Glu-Thr-Asp-Tyr-Tyr-Arg-Lys) were purchased from AnaSpec (San Jose, CA). The c-myc peptide (Cys-Glu-Gln-Lys-Leu-Ile-Ser-Glu-Glu-Asp-Leu) was purchased from Covance (Berkeley, CA). Standard TGF-FLAG peptide (Val-Val-Ala-Ala-Ser-Gln-Lys-Lys-GlnAsp-Tyr-Lys-Asp-Asp-Asp-Asp-Lys-Val-Val) has been described previously [16]. All peptides were used without further purification. Stock solutions were prepared at a concentration of $1 \mu \mathrm{g} / \mu \mathrm{L}$ in HPLC-grade water, and serially diluted with HPLC-grade water.

Immobilized antiphosphotyrosine antibody, avidin agarose, and monoclonal anti-FLAG M2 affinity resin beads were purchased from Sigma Chemical Co., and anti-myc $9 \mathrm{E} 10$ affinity beads were purchased from Covance (Berkeley, CA).

\section{Methods}

Affinity binding for the standard peptides was carried out in compact reaction columns (CRCs) (USB Corporation, Cleveland, $\mathrm{OH}$ ). A typical column volume for a standard was $10 \mu \mathrm{L}$. The beads were washed with 1.5 $\mathrm{mL}$ of $1 \times$ phosphate-buffered saline (PBS) prior to incubation. The peptides were then incubated with the beads for $2 \mathrm{~h}$, in an Eppendorf Thermomixer at $400 \mathrm{rpm}$, at $25^{\circ} \mathrm{C}$. For each standard, a series of different loadings ( $\sim 2$ to 700 pmol) was incubated with the beads. After incubation, the beads were washed with $1.5 \mathrm{~mL}$ of $1 \times$ PBS. The beads were never allowed to dry out.

Details of the TACE (tumor necrosis factor- $\alpha$ converting enzyme) digestion, and isolation of FLAGtagged peptides are described elsewhere [16]. Briefly, the medium concentrate solution was incubated with $\sim 1 \mu \mathrm{g}$ recombinant human TACE extracellular domain [17] at $37{ }^{\circ} \mathrm{C}$ for $4 \mathrm{~h}$, and reactions were stopped by addition of EDTA to $10 \mathrm{mM}$ final concentration. Products containing the FLAG epitope were immunoprecipitated by overnight incubation with anti-FLAG M2 affinity resin in $50 \mathrm{mM}$ Tris $\mathrm{pH} 7.4,150 \mathrm{mM} \mathrm{NaCl}$. The beads were washed five times with $50 \mathrm{mM}$ ammonium bicarbonate, and a $0.5 \mu \mathrm{L}$ aliquot of the settled beads was spotted on the MALDI target.

\section{Mass Spectrometry}

MALDI/TOF-MS was performed on a Bruker Instruments Co. (Billerica, MA) Reflex III, with pulsed ion extraction. MALDI-MS and MALDI-MS/MS were performed on an Applied Biosystems Div. Perkin-Elmer 
Corp. (Foster City, CA) API QSTAR-Pulsar (QSTAR), with argon as the collision gas. The instrument is equipped with a nitrogen laser operating at $337 \mathrm{~nm}$.

For experiments on the Reflex III, recrystallized $\alpha$-cyano-4-hydroxycinnamic acid (HCCA) (Aldrich, Milwaukee, WI) was used as the matrix. The solvent for HCCA was 45:45:10 ethanol:water:formic acid, and used as a saturated solution. A premixed matrix solution containing 2,5-dihydrobenzoic acid (DHB) (Agilent Technologies Inc., Palo Alto, CA) was used without further dilution for experiments on the QSTAR. A 0.5 $\mu \mathrm{L}$ aliquot of the settled beads was spotted on the target, followed by $0.5 \mu \mathrm{L}$ matrix solution, and the solution was allowed to dry at room temperature.

\section{Calculation of the Amount of Peptide on the MALDI Target}

Exactly determining the amount of affinity-bound peptide applied to the MALDI target is difficult. The bed volume of the affinity beads is $10 \mu \mathrm{L}$, and $1 / 20$ of the bed volume is spotted on the MALDI target. However, the entire $0.5 \mu \mathrm{L}$ volume is not solely composed of beads. Between $800-1000$ beads are present in $10 \mu \mathrm{L}$ of beads. However, often only 15-30 beads were present on the MALDI target. Therefore, dividing the amount of peptide incubated with the beads by 25 ( 800 beads incubated divided by 30 beads on target) provides a maximum amount on the MALDI target. Practically, the amount of peptide is most likely much less than what was calculated. This is due to variations in the amount of beads used for immunoprecipitation, as well as the number of beads applied to the MALDI target. For example, if 1000 beads were used for immunoprecipitation, and only 15 were applied to the MALDI target, only $1.5 \%$ of the original peptide solution is present for MS analysis.

\section{Database Searching}

For searching and interpreting MALDI-MS/MS spectra, Mascot software [18] was used. Searches considered only the Homo sapiens genome, and a mass accuracy of $100 \mathrm{ppm}$ in the precursor ion mass and 0.1 $\mathrm{Da}$ in the product ion masses was entered. When applicable, a fixed modification of "Biotin (N-term)" or a variable modification of "Phospho (S,T) and Phospho $(\mathrm{Y})$ " was entered. The nomenclature of Roepstorff and Fohlman [19], later modified by Biemann [20], was used to identify all product ions.

\section{Results and Discussion}

\section{Proof-of-Principle Experiments}

To evaluate the method of direct MALDI-MS/MS sequencing of epitope-containing peptides bound to affinity beads in regards to sensitivity, mass accuracy, and protein identification via database searching and de novo sequencing, model peptides bound to beads containing immobilized anti-phosphotyrosine antibodies, anti-c-myc antibodies and immobilized avidin were analyzed.

\section{Phosphotyrosine/Anti-Phosphotyrosine Binding}

Phosphorylated pKDIR peptide at several concentrations was incubated with an antibody against phosphorylated tyrosine, immobilized on agarose beads. As can be seen in Figure 1a, with a maximum of $234 \mathrm{fmol}$ of affinity-bound peptide loaded on the target, the protonated molecule $\left([\mathrm{M}+\mathrm{H}]^{+}\right)$at $\mathrm{m} / z 1702.553$ of the phosphorylated peptide was observed, with a signal to noise ratio $(\mathrm{S} / \mathrm{N})$ greater than 10 , as well as a loss of 98 $\mathrm{Da}\left(\mathrm{m} / \mathrm{z}\right.$ 1604.477), corresponding to loss of $\mathrm{H}_{3} \mathrm{PO}_{4}$, of approximately $25 \%$ relative intensity. The resolution obtained for the peak at $\mathrm{m} / \mathrm{z} 1702.553$ was 11,601 . The ability of the antibody to completely capture all phosphorylated peptides was examined, by performing MALDI-MS of the pass-through. As shown in Figure 1b, no ion signal is observed at $\mathrm{m} / \mathrm{z} 1702.5$, indicating that the antibody beads have selectively bound all phosphorylated peptide present in the solution.

The MALDI-MS/MS spectrum of $\mathrm{m} / \mathrm{z} 1702.553$ is shown in Figure 1c depicting several $b$ and y ions. The six most abundant product ions from the MALDIMS/MS spectrum were submitted to Mascot for database searching. Because this peptide does not correspond to an enzymatic fragment, no enzyme was specified in the search. Even without any enzyme restriction the insulin receptor protein, along with several homologous proteins, was identified as the top hit, with an average error of $106 \mathrm{ppm}$ in the product ions masses (Note: This mass accuracy could be achieved even from ion signals with $\mathrm{S} / \mathrm{N}=3$ ). Several proteins were identified as the top hit because each protein sequence contains the KDIR peptide. This demonstrates that analyzing peptides affinity-bound to antibody beads does not compromise the mass accuracy of the QqTOF. The ability to search MS/MS spectra, with high mass accuracy, is crucial for the unambiguous identification of peptides [21] and especially for the identification of phosphorylation sites. High mass accuracy is even more critical for this method, since only one peptide, the epitope-containing peptide, is available for protein identification.

This method is not only well suited for testing the specificity of the antibody, as shown in the previous section, but also for testing the selectivity of the antibody. Thus, a mixture of the same KDIR peptide, in both its phosphorylated and its unphosphorylated form, (Figure 2a), was incubated with another aliquot of the antibody beads. For accurate comparison, the same amount of peptide as that shown in Figure 1a was applied to the beads. As can be seen in Figure $2 b$, none of the unphosphorylated sequence was captured by the antibody, indicating that it is highly specific for phosphorylated tyrosine residues. 
(a)

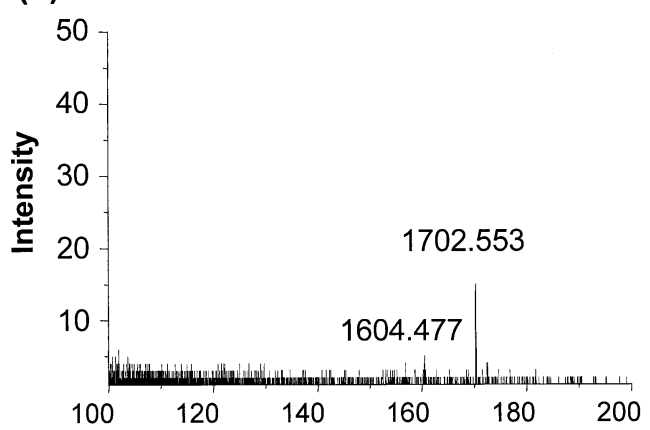

(b)
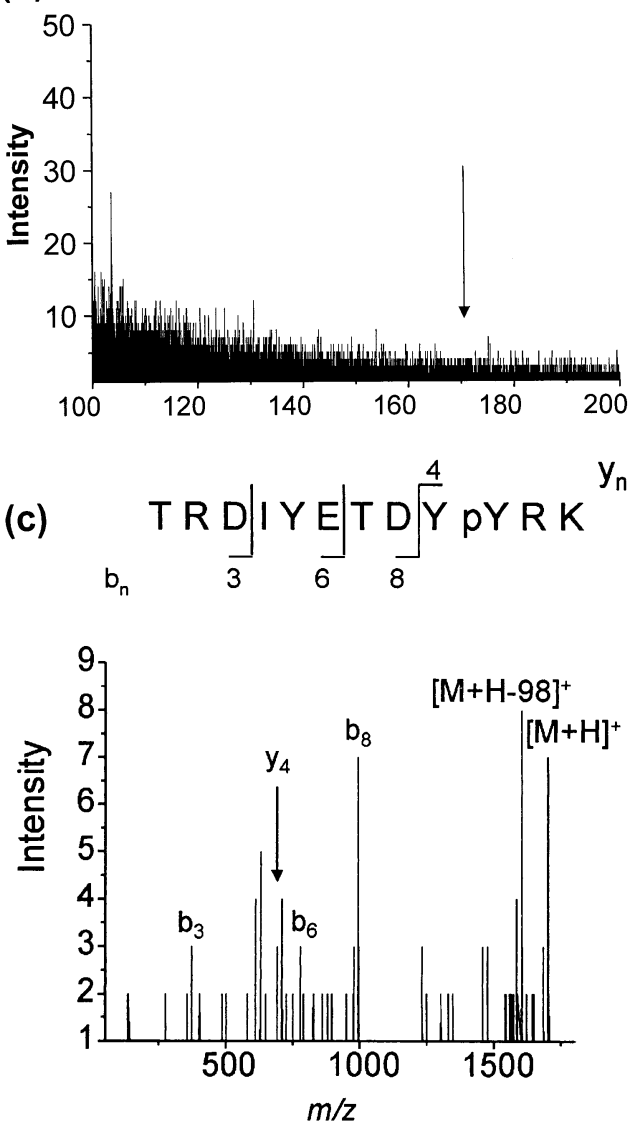

Figure 1. (a) shows the MALDI-MS spectrum of the pKDIR peptide bound to antibody beads. A maximum of $234 \mathrm{fmol}$ is present on the MALDI target. (b) shows the MALDI-MS spectrum of the pass-through, indicating complete capture of the phosphorylated peptide. (c) is the MALDI-MS/MS spectrum of the ion at $\mathrm{m} / \mathrm{z} 1702$.

This has important implications for proteomics, as it demonstrates that anti-phosphotyrosine beads can be used to both isolate and concentrate phosphorylated peptide(s) of interest from a complex mixture. Our previous work with binding this peptide on IMAC beads [14] allows a comparison of the two methods. Using the Fe(III)-IMAC beads, not all of the peptide was removed from the solution, and a significant peptide signal was obtained from MALDI/MS analysis of the pass-through (data not shown). This implies that anti-

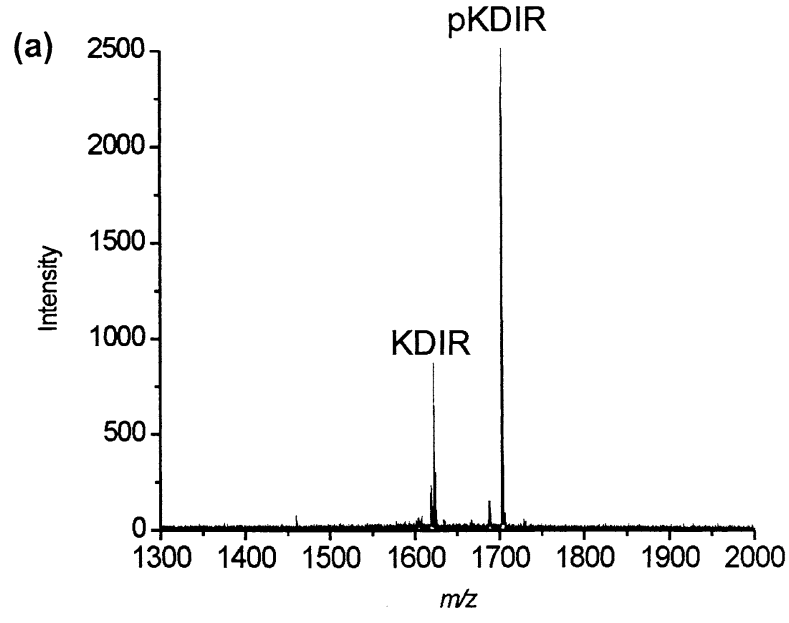

(b)

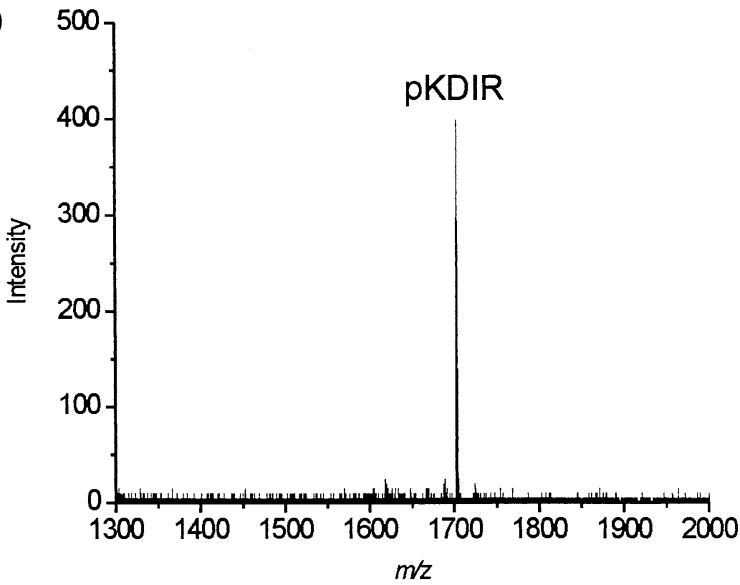

Figure 2. (a) shows the MALDI-MS spectrum of the starting material, an equimolar mixture of phosphorylated and unphosphorylated KDIR peptide. (b) shows the MALDI-MS spectrum of an aliquot of the antibody beads after incubation, indicating that only the phosphorylated sequence has been captured.

phosphotyrosine beads have an advantage over IMAC beads for trapping low levels of phosphopeptide. Unfortunately, however, this advantage only holds for phosphotyrosine-containing phosphopeptides. The binding of serine and threonine phosphopeptides to immobilized anti-phosphoserine and phosphothreonine beads was less successful, and no selectivity was found for phosphoserine and phosphothreonine-containing peptides over their corresponding non-phosphorylated analogues (data not shown). It has been reported that commercially-available antibodies against phosphoserine and phosphothreonine are not sufficient for use in immunoprecipitation [22]. Thus, for peptides phosphorylated on serine or threonine, the IMAC technique would be preferable.

\section{Myc/Anti-Myc Binding}

Several concentrations of a standard peptide containing the $c-m y c$ epitope, from the human $c-m y c$ gene, were incubated with agarose beads containing an immobi- 

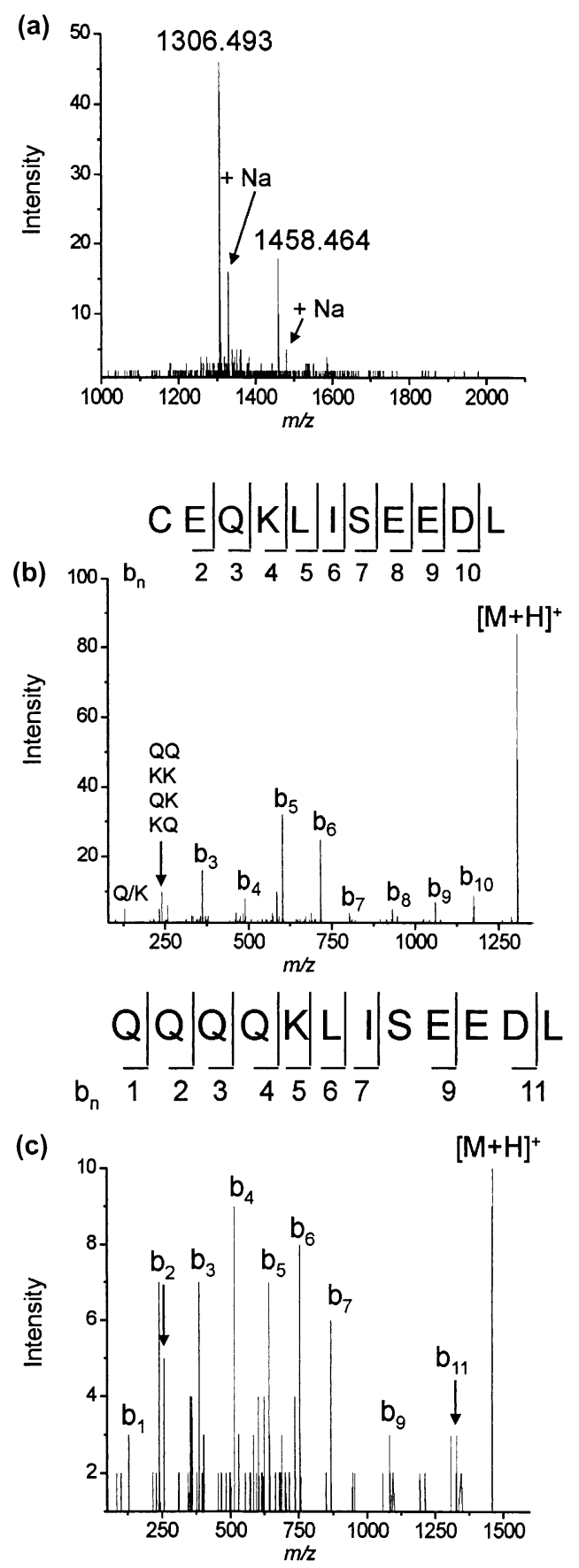

Figure 3. Direct MALDI-MS and MS/MS of the anti-c-myc results. (a) shows the MALDI-MS spectrum of the affinity-captured peptides. The peptide at $\mathrm{m} / \mathrm{z} 1458.464$ was unexpected. (b) shows the MALDI-MS/MS spectrum of the ion at $m / z$ 1306.493. (c) is the MALDI-MS/MS spectrum of the ion at $\mathrm{m} / \mathrm{z} 1458.464$. Note that in both MALDI-MS/MS spectra almost complete series of $b$ ions was observed.

lized antibody against the $c$-myc epitope (EQKLISEEDL). A representative MALDI-MS spectrum is shown in Figure 3a. Although the solution incubated with the affinity beads was supposed to contain only a single peptide sequence, it was apparent from the MALDI-MS spectrum that two peptides containing the $c-m y c$ epitope were present, at $m / z \quad 1306.493$ and 1458.464. A MALDI-MS spectrum of the control anti-c$m y c$ antibody beads confirmed that there was no contamination from the beads at $m / z 1458.464$ (data not shown).

MALDI-MS/MS spectra of these peptides affinitybound to the antibody beads are shown in Figure $3 \mathrm{~b}$ and c. To determine the complete peptide sequence, a slightly higher loading (a maximum of $3.1 \mathrm{pmol}$ ) was applied to the sample target, to increase the number of sequence-specific ions that would be observed. A complete $b$ ion series, from $b_{2}$ to $b_{10}$, was observed for the expected sequence, at $m / z$ 1306.493. Because this peptide is a synthetic construct, and not present in a protein sequence, this MALDI-MS/MS spectrum was not submitted for database searching. The human myc protooncogene protein (accession no. P01106) has a Glu residue $\left(\mathrm{Glu}^{416}\right)$ preceding the epitope tag, instead of a Cys. FASTA (http://www.ebi.ac.uk/fasta33/) was used to search for homologous proteins, and the $c-m y c$ proto-oncogene protein was unambiguously identified. The average mass error observed in these experiments was \pm 85 ppm.

For the unexpected peptide detected in the synthetic standard, de novo sequencing was necessary to identify the amino acid sequence of the peptide. A series of ions, differing in mass by $128 \mathrm{Da}$ was observed, which identified the first four residues of this peptide as Gln, and the fifth residue as Lys. With the high mass accuracy of the QqTOF, Lys (128.094 Da) and Gln (128.059 Da) can be differentiated, even though there is only a $0.04 \mathrm{Da}$ difference. The remaining residues were identical in sequence to the expected peptide. A complete $b$ ion series, from $b_{1}$ to $b_{7}$ was observed, as well as the $b_{9}$ and $b_{11}$ ions. Due to the $b_{8}$ and $b_{10}$ ions not being observed, amino acid pairs were determined from the mass differences measured between the $b_{7}$ and $b_{9}$ ions, as well as the $b_{9}$ and $b_{11}$ ions.

This $c$-myc study emphasizes the importance of being able to rapidly perform MS/MS sequencing of affinitybound peptides. Although the $c-m y c$ epitope has been reported to be (EQKLISEEDL), the peptide at $\mathrm{m} / \mathrm{z}$ 1458.464 only has a portion of this epitope tag (QKLISEEDL), yet was still immunoprecipitated by the anti-c-myc antibody. Partial epitope recognition can lead to cross-reactivity, and isolation of contaminating proteins. It is crucial to be able to quickly identify all proteins isolated by an antibody, especially for protein chips using antibodies. Additionally, this direct MALDI-MS/MS technique simplifies definition of an unknown epitope. Like the previous direct MALDI-MS approach [6], this direct MALDI-MS/MS method is applicable both to the epitope extraction and the epitope excision methodologies.

In the previous method [6], the epitope itself was not sequenced - it stayed affinity bound to the antibody until the MALDI-MS analysis step, and its intact molecular weight was determined by direct MALDI-MS of the affinity beads. Successive iterative enzymatic cleav- 
ages were performed on the affinity-bound intact antibody. This "ladder sequencing" [23] approach allowed one to determine the sequence of the non-epitopicportion of the epitope-containing peptide (residues forming the actual epitope were protected by the antibody and could not be enzymatically cleaved). Enzymatic cleavages from both the N- and C-termini allowed the epitope to be mapped by matching the peptide molecular weight to what was left on the beads after enzymatic cleavage of the non-epitopic portion of the peptide. This approach relied on an antigenic protein with a known sequence.

The ability to sequence the epitope directly opens up the possibility of shortening the process of epitope mapping intact antigens. Getting an enzymatic "sequence tag" of the epitope-containing peptide is no longer a requirement. Instead of a series of successive, iterative, enzymatic digestions and MALDI analyses, it should be possible to use a mix of enzymes and longer digestion times. In this way, one could go directly to the epitope and sequence it with MALDI-MS/MS. This would allow epitope mapping of proteins whose sequence either is not known, or (as in HIV) where the protein sequence is variable. It should also, in principle, be possible to sequence epitopes from unknown proteins, thus identifying both the epitope and the antigenic protein in a single step. One area where this might be of use would be autoimmune diseases. In this case the antibodies could be isolated and immobilized, and their antigenic protein and the epitope could be determined by direct MS/MS analysis of the affinity beads.

This approach would also simplify epitope determination of polyclonal antibodies. While the direct MALDI-MS approach has been demonstrated for a polyclonal antibody with a known 14-residue epitope region [24], all of the bound peptides were digested simultaneously, and sorting out the results can be confusing. (An analogy would be performing Edman sequencing on a mixture of peptides.) This would be very difficult on an unknown epitope. The direct MALDI-MS/MS approach would allow direct sequencing of each of the affinity-bound peptides separately.

Another potential use of this approach is to characterize discontinuous epitopes. In this case, however, the binding to the affinity beads must be performed prior to digestion to avoid structural impairment of the discontinuous epitope which might lead to loss of binding affinity $[25,26]$. In the event that the discontinuous epitope is too high in mass for sequencing by MALDI-MS/MS, the use of higher laser power and/or HCCA instead of DHB can extend the accessible $\mathrm{m} / \mathrm{z}$ range [27]. In addition, the method of dual enzymatic digestion [28] can be used to create smaller affinity-bound peptides with lower MW's amenable to direct sequencing by MALDI-MS/MS.

\section{Biotin/Avidin Binding}

The biotinKDIR peptide was incubated with agarose beads containing an immobilized avidin moiety. Be-
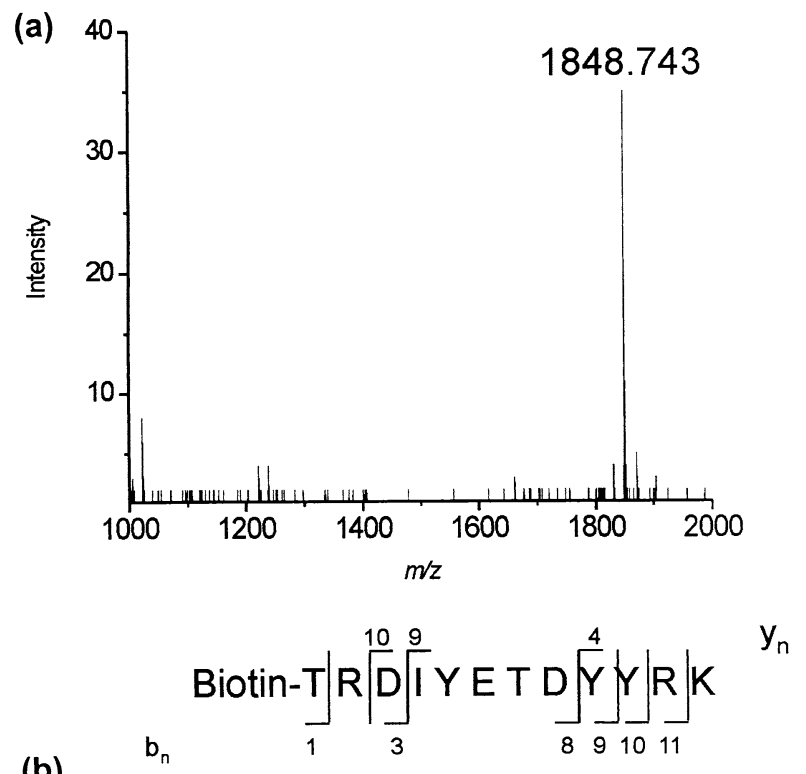

(b)

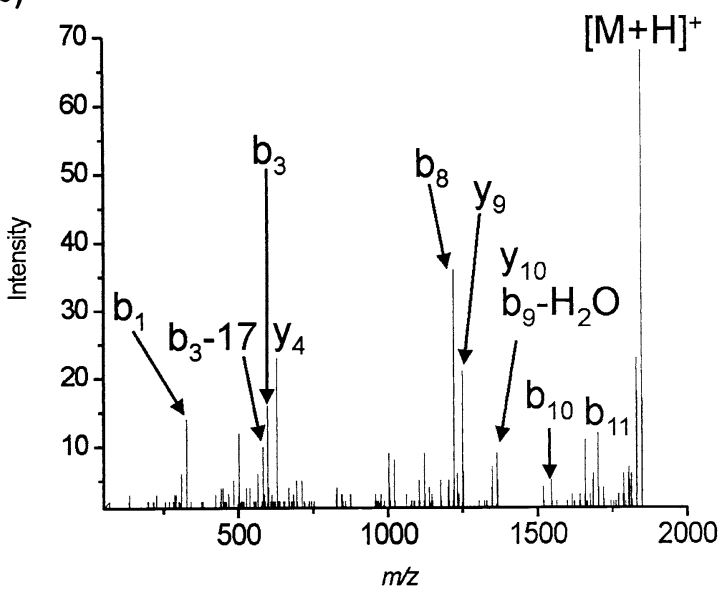

Figure 4. MALDI-MS and MALDI-MS/MS of biotinylated peptide affinity-bound to immobilized avidin beads. (a) is the MALDI-MS spectrum, with a maximum of 2.16 pmol on target. (b) is the MALDI-MS/MS spectrum, with sequence-specific $b$ and $y$ ions indicated.

cause the biotin avidin association is one of the strongest non-covalent interactions, initially an aliquot of beads containing a maximum of 2.16 pmol of affinitybound peptide was placed on the MALDI target, and the MALDI-MS spectrum can be seen in Figure 4a. Strong signal is observed for the protonated molecule $\left([\mathrm{M}+\mathrm{H}]^{+}\right)$, with a $\mathrm{S} / \mathrm{N}$ ratio greater than 30 . MALDIMS/MS was performed on the ion at $m / z 1848.743$, and the MALDI-MS/MS spectrum is shown in Figure $4 \mathrm{~b}$. Several sequence-specific $b$ and y ions were observed, which were sufficient for identifying the peptide sequence.

With a lower sample loading ( $<250 \mathrm{fmol}$ loading), MALDI-MS/MS spectra can still be obtained (Figure 5). Although only four product ions are present with a $\mathrm{S} / \mathrm{N}$ greater than 2, the mass accuracy was high enough to identify the protein by database searching, specifying a fixed modification of $\mathrm{N}$-terminal biotinylation. The in- 

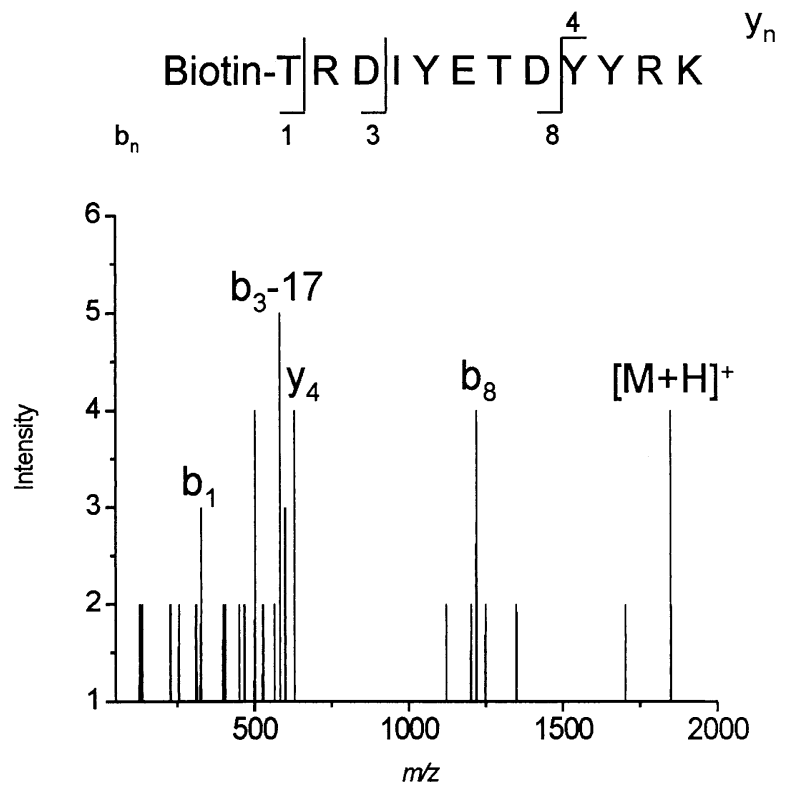

Figure 5. MALDI-MS/MS spectrum of standard biotinylated peptide, affinity-bound to avidin beads, with a maximum of 216 fmol on target.

sulin receptor (accession no. NP_S000199), along with several variants, was identified, with an average error of $65 \mathrm{ppm}$ in the product ion masses. It should be noted that this MALDI-MS/MS spectrum was obtained with a maximum of $216 \mathrm{fmol}$ spotted on the target. Even with mid-femtomole amounts of sample applied to the MALDI target, the protein can unambiguously be identified via database searching.

Although the biotin-avidin association $\left(\mathrm{K}_{\mathrm{d}}=\right.$ $\left.10^{-15} \mathrm{M}\right)[29,30]$ is stronger than the biotin-streptavidin association, $\left(\mathrm{K}_{\mathrm{d}}=10^{-13} \mathrm{M}\right)$ [31] the standard biotinylated peptide was easily dissociated from the avidin agarose under MALDI conditions. Thus, even compounds with high dissociation constants can still be measured using direct MALDI-MS/MS of peptides affinity-bound to antibody beads.

The sequence information obtained on the peptides bound to the affinity beads is very useful for establishing assays to characterize and quantify the binding affinity of the bound peptide, for example, competitive assays with a radioactive labeled derivative of the bound peptide. Thus, the method described in this manuscript makes it possible to obtain the ultimate proof of specific binding.

Nelson and coworkers have performed MALDI/MS analysis directly from BIAcore chips [32-35]. While direct MALDI-MS/MS has not yet been used for the direct analysis of peptides bound to BIAcore chips, the technique described in this manuscript should be applicable to direct MALDI-MS/MS of peptides bound to BIAcore chips as well as affinity beads, thus providing sequence information without the necessity of eluting and concentrating. The combined approach of BIAcore and MALDI-MS/MS would be attractive because the
BIAcore technique provides information on the binding affinity and kinetics which is not provided by MALDIMS/MS.

\section{Are the Peptides Bound to the Antibody Beads?}

There has been some speculation in the literature whether or not the peptides are still bound to the beads following the addition of matrix solution [3, 4]. Quite recently, it was reported that the DHB matrix solvent actually can "elute" the phosphorylated peptides from IMAC beads during the spotting process [3]. To test whether or not the peptides are similarly released from antibody beads during the spotting process, a standard peptide, bound to beads containing an immobilized anti-FLAG antibody, was spotted with HCCA. Spectra were acquired when beads were irradiated by the laser (Figure 6a), and when only matrix crystals were being irradiated by the laser (Figure 6b). Clearly some elution is occurring, as strong signal is obtained when the laser irradiates only matrix crystals. However, it is interesting to note that there is greater signal intensity when the beads are in the laser path, indicating that some peptide may remain bound to, or closely associated with, the antibody beads during the spotting process.

To determine if the acid or the organic solvent was responsible for the release of the peptide from the antibody beads, an aliquot of the beads was spotted using only HCCA and methanol as the matrix solution. The mass spectrum acquired with no beads in the laser path (Figure 6c) indicates that very little peptide was released from the beads. Therefore, it is the addition of acid to the matrix solvent that promotes peptide release from the antibody beads. It has been speculated that the addition of acid to the peptide-antibody complex promotes dissociation [2]. Regardless of whether the peptides remain bound or are eluted, spotting the affinity beads directly on the MALDI target allows detection of mid-femtomole amounts of peptide, with the ability to perform MS/MS analysis.

\section{Application of the Method to Identify In Vitro Proteolysis Products of TACE}

First application of the method has been performed to assess the ability of tumor necrosis factor- $\alpha$ converting enzyme (TACE) to cleave a membrane-proximal site in pro-transforming growth factor- $\alpha$ (proTGF $\alpha$ ). TGF $\alpha$ is a member of the epidermal growth factor (EGF) family [36]. TGF $\alpha$ is synthesized as a membrane bound precursor ( $\operatorname{proTGF} \alpha$ ) that is proteolytically cleaved to release the EGF receptor binding ligand. Until recently, the identities of the enzymes required for proteolytic processing were unknown. A novel protease, tumor necrosis factor- $\alpha$ converting enzyme (TACE), was identified as being responsible for converting the membrane-bound pro-TGF $\alpha$ to its soluble form [16]. A soluble epitope-tagged version of the ectodomain of 

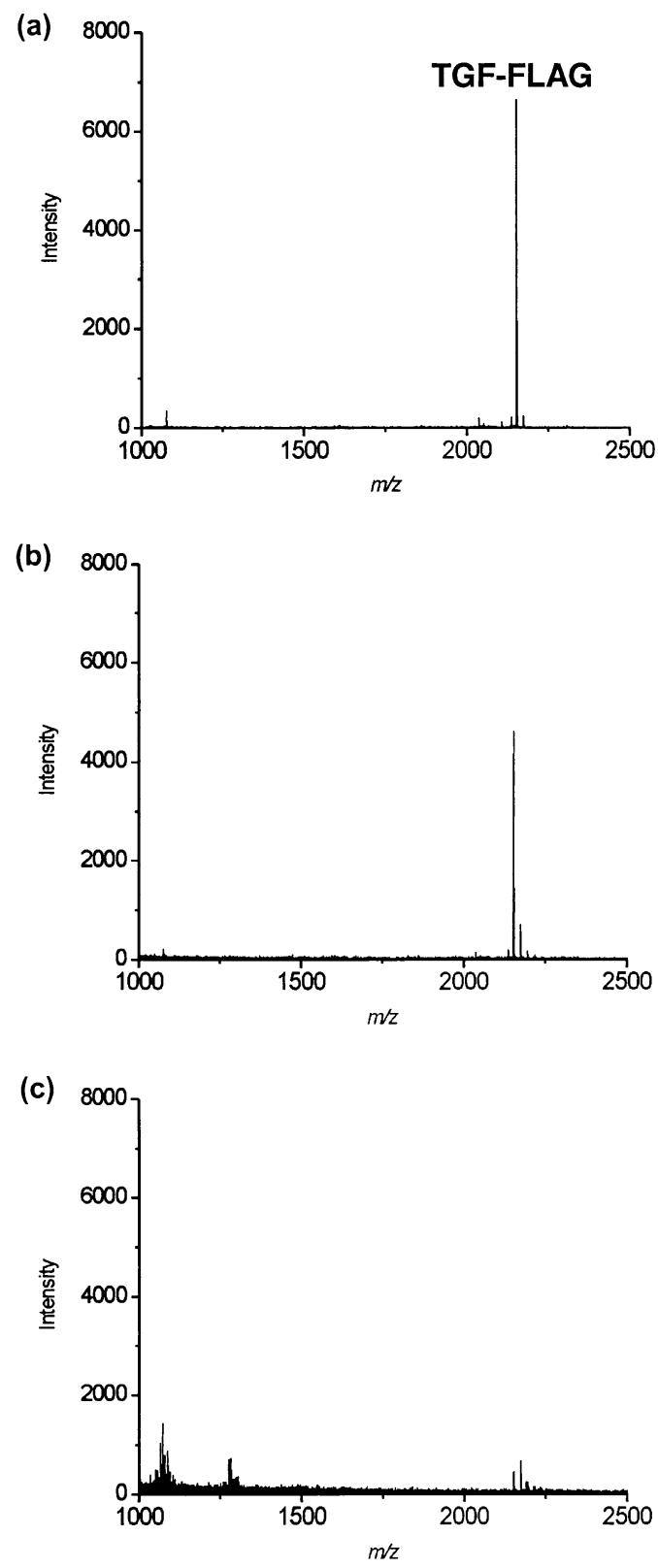

Figure 6. MALDI-MS spectrum of standard TGF-FLAG peptide. (a) was acquired with affinity beads irradiated by laser. (b) was acquired with only matrix crystals irradiated. The matrix solvent used for (a) and (b) was $45 \%$ ethanol, $45 \%$ water, and 10\% formic acid. (c) was acquired with only matrix crystals irradiated, with methanol as the matrix solvent.

proTGF $\alpha$, (proTGFecto) was created with a C-terminal FLAG tag [37] (DYKDDDDKVV) [16]. Media concentrate solution from cells expressing this construct was incubated with recombinant human TACE extracellular domain for $4 \mathrm{~h}$ at $37^{\circ} \mathrm{C}$. Anti-FLAG M2 affinity resin was subsequently added at the end of the incubation. Immunoprecipitates were washed with $50 \mathrm{mM}$ ammonium bicarbonate and a small aliquot of the beads was directly placed on the MALDI target.

Two peaks were observed in the MALDI-TOF/MS spectrum, at $\mathrm{m} / \mathrm{z} 2151.447$ and 1811.119 , as seen in (a)

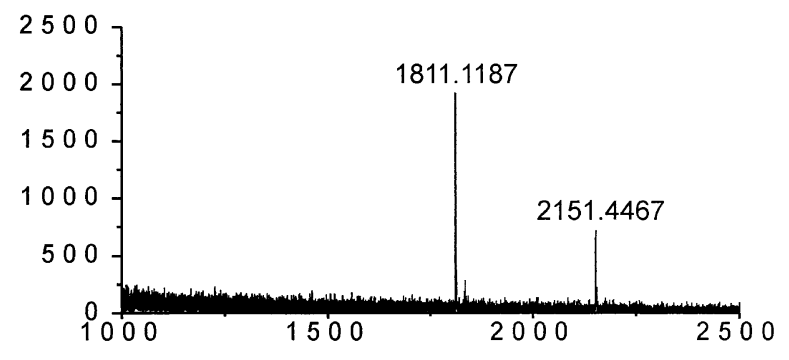

(b)

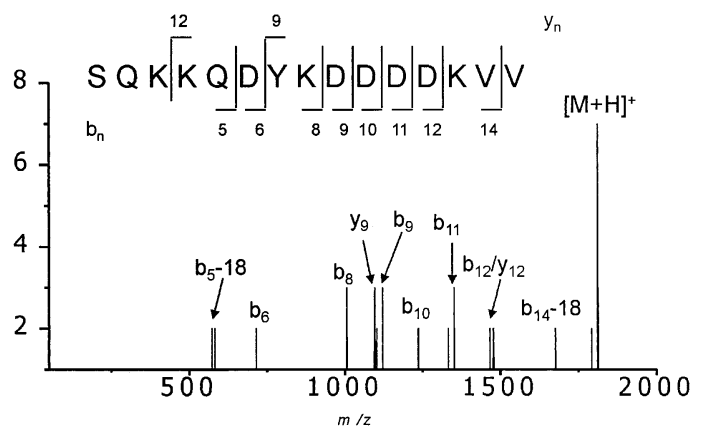

Figure 7. Direct MALDI-MS and MALDI-MS/MS spectra of the FLAG-tagged TGFecto peptides cleaved by TACE. (a) is the MALDI-MS spectrum. The peptide at $\mathrm{m} / \mathrm{z} 1811.119$ was unexpected. (b) is the MALDI-MS/MS spectrum of the ion at $\mathrm{m} / \mathrm{z}$ 1811.199.

Figure 7a. The peak at 2151.447 matched the molecular weight of a synthetic peptide with the sequence of the expected product, VVAASQKKQDYKDDDDKVV with cleavage occurring between $\mathrm{Ala}^{89}$ and $\mathrm{Val}^{90}(\mathrm{~m} / \mathrm{z}$ 2151.350) [16]. Because the second product (at $\mathrm{m} / \mathrm{z}$ 1811.119) was unexpected, its sequence was determined by MALDI-MS/MS.

The product ion spectrum of $m / z 1811.119$ is shown in Figure $7 \mathrm{~b}$. Although the overall intensity was low, a series of $b$ ions confirmed that the sequence of the peptide was SQKKQDYKDDDDKVV, thus confirming a second in vitro TACE cleavage site on proTGFecto, between $\mathrm{Ala}^{94}$ and $\mathrm{Ser}^{95}$. Determination of the sequence of the $\mathrm{m} / \mathrm{z} 1811$ product, therefore, allowed unambiguous confirmation of the identity of an unexpected proteolytic product, thereby providing firm indication of a second, specific cleavage event.

\section{Conclusions}

In this study, we report a powerful method for isolation, identification, and sequencing of peptides bound to immobilized antibodies on affinity beads. Placing an aliquot of the affinity beads directly on the MALDI target eliminates sample loss that can occur with peptide elution followed by nESI-MS/MS, which is the usual "method of choice" for peptide sequencing. Direct on-target analysis of affinity-bound analytes is also a much more rapid approach than the previously-used MALDI-MS based technique involving enzymatic ladder sequencing of affinity-bound peptides [6]. It should 
also allow epitope mapping of proteins with unknown or variable sequences, and should greatly simplify the epitope mapping of polyclonal antibodies.

This new method, which provides sample enrichment with minimal sample handling, has been demonstrated for a variety of antibody beads commonly used in protein purification studies, and has been used to identify an unknown cleavage site on a FLAG-tagged protein. All affinity techniques, including this method, are vulnerable to non-specific binding. The ability to obtain sequence information on the affinity-bound peptides makes it easier to distinguish between specificallyand non-specifically-bound peptides, especially when the affinity tag is known, as it is in the FLAG peptide example described here.

Nelson et al. [32-35], have described the use of epitope-tagged proteins and peptides in direct BIAcoreMALDI-MS analysis. The applications of the direct MALDI MS/MS would be analogous, with the important advantage that it would not rely on incomplete cleavage of the protein and analysis by peptide mass fingerprinting or enzymatic protein ladder sequencing in order to identify the protein. Using MALDI-MS/MS, a protein (or a family of homologous proteins) could be identified from a single bound peptide.

In addition, this approach is also applicable to the characterization of non-epitope tagged proteins. This has been demonstrated for several proteins, including cancer-related proteins like p53, and is currently being used for the development of a mass spectrometricbased protein chip [38] for rapid determination of differential expression of targeted proteins.

The high mass accuracy of the QqTOF mass spectrometer is not affected by the direct MALDI analysis of affinity-bound peptides beads. With this high mass accuracy, commercially-available proteomics software packages such as Mascot can identify a protein (or a family of homologous proteins which contain the same peptide) from the MS/MS spectrum of one of its peptides, and can automatically localize the modification to a specific amino acid within that peptide, even when the peptide is affinity-tagged. Thus, the method is well suited for molecular characterization of epitopecontaining peptides, and for protein chips.

\section{Acknowledgments}

This work was supported by a gift from an anonymous donor for research targeted to Proteomics and Cystic Fibrosis, and by grant CA85410 (to DCL) from the National Institutes of Health.

\section{References}

1. Suckau, D.; Kohl, J.; Karwath, G.; Schneider, K.; Casaretto, M.; Bitter-Suermann, D.; Przybylski, M. Molecular Epitope Identification by Limited Proteolysis of an Immobilized AntigenAntibody Complex and Mass Spectrometric Peptide Mapping. Proc. Natl. Acad. Sci. U.S.A. 1990, 87, 9848-9852.

2. Kiselar, J. G.; Downard, K. M. Direct Identification of Protein Epitopes by Mass Spectrometry without Immobilization of
Antibody and Isolation of Antibody-Peptide Complexes. Anal. Chem. 1999, 71, 1792-1801.

3. Hart, S. R.; Waterfield, M. D.; Burlingame, A. L.; Cramer, R. Factors Governing the Solubilization of Phosphopeptides Retained on Ferric NTA IMAC Beads and Their Analysis by MALDI TOFMS. J. Am. Soc. Mass Spectrom. 2002, 13, 1042 1051.

4. Papac, D. I.; Hoyes, J.; Tomer, K. B. Direct Analysis of Affinity-Bound Analytes by MALDI/TOF MS. Anal. Chem. 1994, 66, 2609-2613.

5. Gevaert, K.; Demol, H.; Puype, M.; Broekaert, D.; De Boeck, S.; Houthaeve, T.; Vandekerckhove, J. Peptides Adsorbed on Reverse-Phase Chromatographic Beads as Target for Femtomole Sequencing by Post-Source Decay Matrix-Assisted Laser Desorption/Ionization-Reflectron Time-of-Flight Mass Spectrometry (MALDI-RETOF-MS). Electrophoresis 1997, 18, 2950 2960.

6. Parker, C. E.; Tomer, K. B. MALDI/MS-Based Epitope Mapping of Antigens Bound to Immobilized Antibodies. Mol. Biotechnol. 2002, 20, 49-62.

7. Qian, X.; Zhou, W.; Khaledi, M. G.; Tomer, K. B. Sequential Cleavages of Peptides and Proteins Affinity-Bound to Immobilized Metal Ion Beads by Matrix-Assisted Laser Desorption/ Ionization Mass Spectrometry. Anal. Biochem. 1999, 274, 174180.

8. Parker, C. E.; Tomer, K. B. Epitope Mapping by a Combination of Epitope Excision and MALDI-MS. Methods Mol. Biol. 2000, 146, 185-201.

9. Zhao, Y.; Chait, B. T. Protein Epitope Mapping by Mass Spectrometry. Anal. Chem. 1994, 66, 3723-3726.

10. Parker, C. E.; Papac, D. I.; Tomer, K. B. Monitoring Cleavage of Fusion Proteins by Matrix-Assisted Laser Desorption/Ionization Mass Spectrometry: Recombinant HIV-1IIIB p26. Anal. Biochem. 1996, 239, 25-34.

11. Parker, C. E.; Papac, D. I.; Trojak, S. K.; Tomer, K. B. Epitope Mapping by Mass Spectrometry: Determination of an Epitope on HIV-1 IIIB p26 Recognized by a Monoclonal Antibody. J. Immunol. 1996, 157, 198-206.

12. Loboda, A. V.; Krutchinsky, A. N.; Bromirski, M.; Ens, W.; Standing, K. G. A Tandem Quadrupole/Time-of-Flight Mass Spectrometer with a Matrix-Assisted Laser Desorption/Ionization Source: Design and Performance. Rapid Commun. Mass Spectrom. 2000, 14, 1047-1057.

13. Baldwin, M. A.; Medzihradszky, K. F.; Lock, C. M.; Fisher, B.; Settineri, T. A.; Burlingame, A. L. Matrix-Assisted Laser Desorption/Ionization Coupled with Quadrupole/Orthogonal Acceleration Time-of-Flight Mass Spectrometry for Protein Discovery, Identification, and Structural Analysis. Anal. Chem. 2001, 73, 1707-1720.

14. Raska, C. S.; Parker, C. E.; Dominski, Z.; Marzluff, W. F.; Glish, G. L.; Pope, R. M.; Borchers, C. H. Direct MALDI-MS/MS of Phosphopeptides Affinity-Bound to IMAC Beads. Anal. Chem. 2002, 74, 3429-3433.

15. Wang, H.; Tseng, K.; Lebrilla, C. B. A General Method for Producing Bioaffinity MALDI Probes. Anal. Chem. 1999, 71, 2014-2020.

16. Sunnarborg, S. W.; Hinkle, C. L.; Stevenson, M.; Russell, W. E.; Raska, C. S.; Peschon, J. J.; Castner, B. J.; Gerhart, M. J.; Paxton, R. J.; Black, R. A.; Lee, D. C. Tumor Necrosis Factor- $\alpha$ Converting Enzyme (TACE) Regulates Epidermal Growth Factor Receptor Ligand Availability. J. Biol. Chem. 2002, 277, $12838-12845$

17. Doedens, J. R.; Black, R. A. Stimulation-Induced Down-Regulation of Tumor Necrosis Factor- $\alpha$ Converting Enzyme. J. Biol. Chem. 2000, 275, 14598-14607.

18. Perkins, D. N.; Pappin, D. J.; Creasy, D. M.; Cottrell, J. S. Probability-Based Protein Identification by Searching Se- 
quence Databases Using Mass Spectrometry Data. Electrophoresis 1999, 20, 3551-3567.

19. Roepstorff, P.; Fohlman, J. Proposal for a Common Nomenclature for Sequence Ions in Mass Spectra of Peptides. Biomed. Mass Spectrom. 1984, 11, 601.

20. Biemann, K. Contributions of Mass Spectrometry to Peptide and Protein Structure. Biomed. Environ. Mass Spectrom. 1988, 16, 99-111.

21. Clauser, K. R.; Baker, P.; Burlingame, A. L. Role of Accurate Mass Measurement $( \pm 10 \mathrm{ppm})$ in Protein Identification Strategies Employing MS or MS/MS and Database Searching. Anal. Chem. 1999, 71, 2871-2882.

22. McLachlin, D. T.; Chait, B. T. Analysis of Phosphorylated Proteins and Peptides by Mass Spectrometry. Curr. Op. Chem. Bio. 2001, 5, 591-602.

23. Chait, B. T.; Wang, R.; Beavis, R. C.; Kent, S. B. H. Protein Ladder Sequencing. Science 1993, 262, 89-92.

24. Jeyarajah, S.; Parker, C. E.; Sumner, M. T.; Tomer, K. B. Matrix-Assisted Laser Desorption/Ionization Mass Spectrometry Mapping of Human Immunodeficiency Virus-gp120 Epitopes Recognized by a Limited Polyclonal Antibody. J. Am. Soc. Mass Spectrom. 1998, 9, 157-165.

25. Hochleitner, E. O.; Borchers, C.; Parker, C.; Bienstock, R. J.; Tomer, K. B. Characterization of a Discontinuous Epitope of the Human Immunodeficiency Virus (HIV) Core Protein p24 by Epitope Excision and Differential Chemical Modification Followed by Mass Spectrometric Peptide Mapping Analysis. Protein Science 2000, 9, 487-496.

26. Hochleitner, E. O.; Gorny, M. K.; Zolla-Pazner, S.; Tomer, K. B. Mass Spectrometric Characterization of a Discontinuous Epitope of the HIV Envelope Protein HIV-gp120 Recognized by the Human Monoclonal Antibody 1331A. J. Immunol. 2000, 164, 4156-4161.

27. Raska, C. S.; Parker, C. E.; Huang, C.; Han, J.; Glish, G. L.; Pope, M.; Borchers, C. H. Pseudo-MS3 in a MALDI Orthogonal Quadrupole Time-of-Flight Mass Spectrometer. J. Am. Soc. Mass Spectrom. 2002, 13, 1034-1041.
28. Han, J.; Pope, M.; Borchers, C.; Graves, L. M. Mapping of Protein Phosphorylation by Dual Enzyme Digestion and Matrix-Assisted Laser Desorption/Ionization-Quadrupole Orthogonal Time-of-Flight Mass Spectrometry. Anal. Biochem. 2002, 310, 215-218.

29. Green, N. M. Avidin. Adv. Prot. Chem. 1975, 29, 85-133.

30. Kohanski, R.A.; Lane, M. D. Monovalent Avidin Afinity Columns. Methods Enzymol. 1990, 184, (Avidin-Biotin Technol.) 194-200.

31. Piran, U.; Riordan, W. J. Dissociation Rate Constant of the Biotin-Streptavidin Complex. J. Immunol. Methods 1990, 133, 141-143.

32. Krone, J. R.; Nelson, R. W.; Dogruel, D.; Williams, P.; Granzow, R. BIA/MS: Interfacing Biomolecular Interaction Analysis with Mass Spectrometry. Anal. Biochem. 1997, 244, 124-132.

33. Nelson, R. W.; Krone, J. R.; Dogruel, D.; Tubbs, K.; Granzow, R.; Jansson, O. Interfacing Biomolecular Interaction Analysis with Mass Spectrometry and the Use of Bioreactive Mass Spectrometer Probe Tips in Protein Characterization. Techniques in Protein Chemistry VIII. Proceedings of the Symposium of the 10th Protein Society; San Jose, CA, August, 1996 (1997); pp 493-504.

34. Nelson, R. W.; Krone, J. R.; Jansson, O. Surface Plasmon Resonance Biomolecular Interaction Analysis Mass Spectrometry. 1. Chip-Based Analysis. Anal. Chem. 1997, 69, 43634368.

35. Nelson, R. W.; Jarvik, J. W.; Taillon, B. E.; Kemmons, K. A. BIA/MS of Epitope-Tagged Peptides Directly from E. coli Lysate: Multiplex Detection and Protein Identification at LowFemtomole to Subfemtomole Levels. Anal. Chem. 1999, 71, $2858-2865$.

36. Lee, D. C.; Fenton, S. E.; Berkowitz, E. A.; Hissong, M. A. Transforming Growth Factor- $\alpha$ : Expression, Regulation, and Biological Activities. Pharm. Res. 1995, 47, 51-85.

37. Einhauer, A.; Jungbauer, A. The FLAG Peptide, a Versatile Fusion Tag for the Purification of Recombinant Proteins. J. Biochem. Biophys. Methods 2001, 49, 455-465.

38. Borchers, C. H. (patent application filed on 12/02/2002); U.S. Patent Application Pub. 2002. 